\title{
Utilidad diagnóstica de la ecografía de vaina de nervio óptico (EVNO), como método no invasivo para la detección de hipertension intracraneal. Estudio prospectivo de 95 mediciones comparado con monitoreo invasivo en Chile Diagnostic utility of the optic nerve sheath diameter (ONSD), as a non-invasive method for the detection of intracranial hypertension. Prospective study of 95 measurements com- pared with invasive monitoring in Chile
}

\author{
Julio García Molina ${ }^{1}$ \\ Neurocirujano, Hospital Puerto Montt.
}

Rev. Chil. Neurocirugía 45: 38-44, 2019

Resumen

Introducción: El síndrome de hipertensión endocraneal (HTEC) es una condición grave con riesgo vital, causada por un gran número de enfermedades neurológicas y sistémicas. El examen de referencia para su diagnóstico, es la medición invasiva de la presión intracraneana (PIC). Nuestra hipótesis es que la ecografía de vaina de nervio óptico es una herramienta útil como método no invasivo en la detección de HTEC, comparado con el método de referencia actual. Material y Método: Estudio de recolección prospectiva de datos secundarios anonimizados, sobre el valor de la EVNO enmascarado al valor de la PIC por drenaje ventricular externo (DVE), realizado por operador único en pacientes adultos del Hospital Puerto Montt entre 2015 y 2017. Resultados: Se incluyeron 59 pacientes con un total de 95 mediciones. Se obtuvo una sensibilidad $88 \%$, especificidad del $92 \%$ con una valor predictivo positivo (VPP) y negativo (VPN) de $85 \%$ y $94 \%$ respectivamente. La curva de ROC nos muestra un área bajo la curva de 0,95. Discusión: Los resultados obtenidos son comparables a los reportados en la literatura, avalando ampliamente la utilidad de esta técnica no invasiva. Este constituye el primer trabajo reportado en Chile para la validación y difusión de dicha técnica ecográfica en nuestra realidad local. Conclusión: La EVNO es una técnica accesible, sensible y específica para la detección de HTEC de manera no invasiva. Su potencial es evidente más allá del neurointensivo, pero debe utilizarse como herramienta de apoyo y no reemplazar el monitoreo invasivo cuando este se encuentre indicado.

Palabras clave: Hipertensión intracranial, diámetro vaina de nervio óptico, ultrasonido, ecografía, sonografía, monitoreo no invasivo de presión intracraneal.

\begin{abstract}
Introduction: The endocranial hypertension syndrome (HTEC) is a serious condition with life risk, caused by a large number of neurological and systemic diseases. The reference test for its diagnosis is the invasive measurement of intracranial pressure (PIC). Our hypothesis is that the optic nerve sheath ultrasound is a useful tool as a non-invasive method in the detection of HTEC, compared with the current reference method. Material and Method: Prospective study of anonymized secondary data on the value of the EVNO masked to the value of the PIC by external ventricular drainage (DVE), performed by single operator in adult patients of the Hospital Puerto Montt, between 2015 and 2017. Results: 59 patients were included with a total of 95 measurements. A sensitivity of $88 \%$, specificity of $92 \%$ was obtained with a positive predictive value (PPV) and a negative
\end{abstract}


value (NPV) of $85 \%$ and $94 \%$ respectively. The ROC curve shows an area under the curve of 0.95 . Discussion: The results obtained are comparable to those reported in the literature, widely supporting the utility of this non-invasive technique. This is the first work reported in Chile for the validation and dissemination of this ultrasound technique in our local reality. Conclusion: EVNO is an accessible, sensitive and specific technique for the detection of HTEC in a non-invasive way. Its potential is evident beyond the neurointensive, but it should be used as a support tool and not replace invasive monitoring when it is indicated.

Key words: Intracranial hypertension, optic nerve sheath diameter, ultrasonography, ecography, sonography, non-invasive intracranial pressure monitoring.

\section{Introducción}

El síndrome de hipertensión endocraneal (HTEC) es una condición grave con riesgo vital, causada por un gran número de enfermedades neurológicas y sistémicas. Si no es detectada y manejada a tiempo, puede llevar a daño neurológico irreversible o la muerte ${ }^{1,2}$. Actualmente, el examen de referencia para su diagnóstico, es medición cuantitativa de la presión intracraneana (PIC), a través de un catéter intraventricular ${ }^{3,4}$. Esto se realiza mediante un procedimiento invasivo, que implica la realización de una craneotomía. Las complicaciones asociadas a esta intervención incluyen hemorragias 1,1\% a $5,8 \%{ }^{3,5}$ infección $8,8 \%{ }^{6}$, disfunción $6,3 \%^{3}$ y mala posición en un $3 \%{ }^{3}$. Las limitaciones de esta técnica son: técnica invasiva, solo aplicable en unidades de paciente crítico, disponer de un neurocirujano, contar con el equipamiento adecuado, el costo de insumos, así como estar contraindicado en pacientes que cursen con coagulopatías o trombocitopenia 7 .

A la fecha se han postulado múltiples técnicas no invasivas para la estimación de la PIC, entre las cuales podemos mencionar la ultrasonografía doppler transcraneal, desplazamiento de la membrana timpánica, potenciales visuales evocados, oftalmodinamometría, pupilometría, tomografía de escaneo laser confocal de nervio óptico, tomografía de coherencia óptica ${ }^{4,8}$. Si bien la mayoría de estas pruebas ha sido relacionada con una elevación de la PIC, ninguna de estas ha logrado aceptación en la práctica clínica por diversas limitaciones, tanto técnicas y económicas, así como validación ${ }^{4,8,9}$.

A diferencia de la anteriores, la medición mediante ecografía de diámetro de la vaina del nervio óptico (EVNO), es una técnica prometedora y muy estudiada, que posee certeza diagnostica reportada en 2 meta-análisis de adultos con trauma encefalocraneano (TEC) y hemorragia intracerebral (HIC). En ellos se analizan 6 estudios prospectivos ciegos observacionales con un total de 249 y 231 pacientes, en los que se compara la medición invasiva de la PIC con EVNO ${ }^{10-15}$; los resultados publicados corresponden a un $87 \%$ y $90 \%$ de sensibilidad, $80 \%$ y $85 \%$ de especificidad, con un odds ratio de 25 y 51 respectivamente ${ }^{7,9}$.

La reproducibilidad de la técnica cuenta con respaldo en la literatura, en el metaanálisis realizado por Dubourg ${ }^{7}$ describe una mediana de variabilidad intraobservador e interobservador de $0,2 \mathrm{~mm}$ y 0,2 a $0,3 \mathrm{~mm}$ respectivamente, siendo ratificada su reproducibilidad por Bäuerle ${ }^{16,17}$ y Ballantyne ${ }^{18}$.

Son destacables otras ventajas de esta técnica, que además no ser invasiva, es de rápida realización y fácil entrenamiento, bajo costo y amplia disponibilidad, incluso en centros donde no se cuenta con tomografía o neurocirujano, por lo cual posee potencial para ser utilizada en servicios de urgencia ${ }^{19}$.

Lo anterior pudo ser corroborado en nuestro centro, en una experiencia inicial presentada en el año 2015 con 6 pacientes y 18 mediciones de EVNO en presencia de un DVE para monitoreo de $\mathrm{PIC}$, y el seguimiento ecográfico de un caso de HTEC por hidrocefalia obstructiva.

Nuestra hipótesis alterna $\left(\mathrm{H}_{1}\right)$ es que la EVNO tiene la suficiente sensibilidad y especificidad para ser útil en la detección de HTEC, comparado con el método de referencia actual (DVE).

\section{Materiales y Métodos}

Se realizó un estudio de recolección prospectiva de datos anomizados sobre el valor de EVNO, enmascarado al valor de la PIC mediante monitoreo invasivo por DVE, en el Hospital Base de Puerto Montt, Chile. En el período de estudio comprendido entre agosto de 2015 a agosto de 2017. Se incluyeron pacientes con diversa patología intracraneal con indicación explicita de monitoreo de PIC para su manejo. Las mediciones ecográficas consignadas fueron realizadas por un único operador, sujeto a la disponibilidad de clínico. El operador ecográfico fue un neurocirujano con entrenamiento en ecografía ocular y un año previo de experiencia en ella. Al momento de la realización del examen, se ocultaba el valor del monitoreo de PIC al operador (rotando el monitor), el cual era entregado al finalizar el procedimiento. El punto de corte utilizado para considerar un examen como positivo es mayor de $5,5 \mathrm{~mm}$; el valor considerado para hipertensión intracraneal es mayor de 20 mmhg por DVE.

\section{Criterios de Inclusión:}

1. Mayores de 18 años.

2. Sin patología ocular previa o aguda.

3. Consentimiento informado del paciente o familiar.

4. Necesidad de monitoreo de PIC mediante DVE debido a patología intracraneal.

\section{Criterios Exclusión:}

1. Menores de 18 años.

2. Patología ocular previa o aguda (se incluyen grandes equimosis orbitarias).

3. Rechazo de procedimiento.

4. Falta de disponibilidad del operador.

\section{La técnica ecográfica utilizada se detalla a continuación: i) Especificaciones técnicas del equipo}

De acuerdo a la literatura actual, para la visualización de la vaina del nervio óptico (VNO), se requiere de un transductor lineal con una frecuencia superior a 7,5 mHz $\mathrm{mH}^{7,9,25}$ con una insonación programada entre 4 a $5 \mathrm{~cm}$ de profundidad, habitualmente predeterminado 
como "partes pequeñas" o "superficial"'.

En nuestro afán de seguir las normas internacionales, consideramos como parámetros norma en la realización de ultrasonido ocular, las recomendaciones realizadas por la "US Food and Drug and Drug Administration" (FDA) y la "World Federation for Ultrasound in Medicine and Biology (WFUNB), definidos con "termal index" o $\mathrm{TI}<1,0$ y "mechanical index" o $\mathrm{Ml}<0,23^{26,27}$.

Para nuestra investigación contamos con el equipo "Sistema ultrasonido General Electric modelo LOGIQ e", transductor "H40420LY 12RL-RS Lineal" con rango de frecuencia 5,0 a $13 \mathrm{Mhz}$, que cumple con los requerimientos previamente mencionados.

\section{ii) Técnica ecográfica}

Para la realización sonografía transbulbar con ojo cerrado, utilizaremos la técnica más recomendada y utilizada en la literatura ${ }^{19,25,28,29}$, la cual detallamos a continuación.

\section{Descripción de la técnica:}

1. Paciente en decúbito supino, de preferencia cabecera en $30^{\circ}$.

2. Ojo cerrado, de preferencia con mirada central como guía de orientación. - En pacientes con compromiso de conciencia, se utiliza tela de papel en el reborde del parpado superior, a fin de mantener el ojo cerrado y evitar artefactos. Siguiendo el eje ocular en relación a la posición pupilar.

3. Aplicar gel ecográfico no alcohólico a transductor

4. Posicionar transductor suavemente en la porción temporal del parpado superior, con orientación perpendicular al eje vertical y una angulación en torno a $30^{\circ}$, en el plano horizontal (Figura 1).

5. Se procede a reconocer las estructuras orbitarias y oculares (Figura 2)

6. Identificar el disco óptico como una banda hiperecogénica.

7. Visualizar la VNO proyectada hacia posterior, en relación a disco óptico, como una estructura hiperecogénica rodeada de una delgada banda hiperecogénica conformada por su cobertura meníngea, y resto de tejido hiperecogénico periférico, que corresponde a tejido graso periocular.

8. Realizar una primera medición de 3 $\mathrm{mm}$, perpendicular al eje mayor de la VNO, desde la porción posterior de la retina.

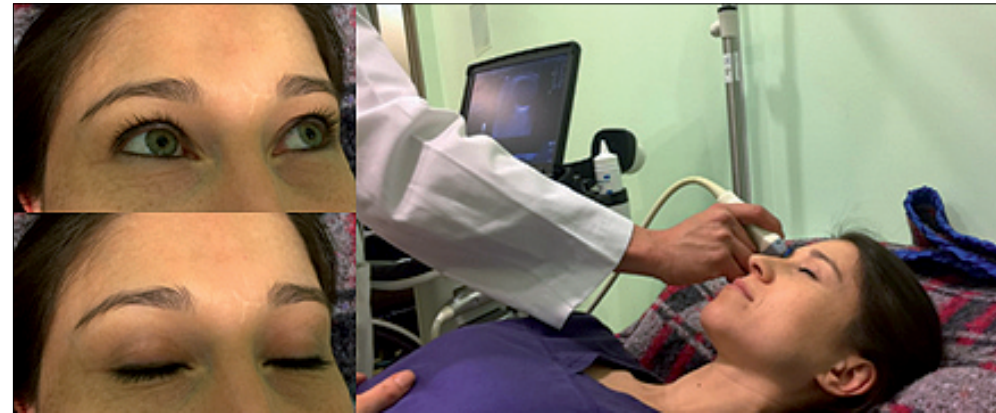

Figura 1. Técnica ecográfica sobre ojo cerrado.

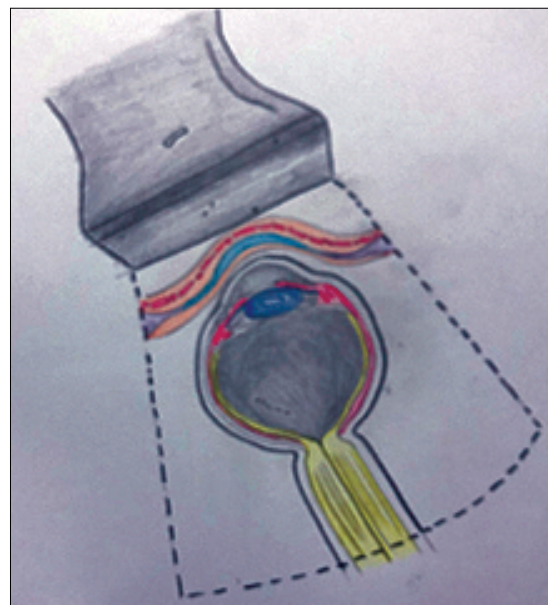

Figura 2. Diagrama de la evaluación sonográfica del ojo y vaina de nervio óptico, con ojo cerrado.

9. Una segunda medición perpendicular a la primera, entre los limites hiperecogénicos de la VNO, configurando su diámetro (Figura 3 ).

10. Repetir la medición en 3 oportunidades, considerando como valor final el promedio de ellas.

11. Se debe realizar el mismo procedimiento en cada ojo.

\section{iii) Riesgos y precauciones especia-} les

Por tratarse de una técnica no invasiva, lo riesgos son mínimos, principalmente de carácter local en relación a la presión excesiva sobre el globo ocular con el transductor y el contacto de gel ecográfico con la conjuntiva, generando irritación química local. Para reducir esto hemos optado por utilizar gel libre de alcohol, y protocolarmente consultar en reiteradas oportunidades sobre la comodidad del participante, con énfasis en solo un contacto sutil contra el globo ocular.

Se excluyen los pacientes con patolo-

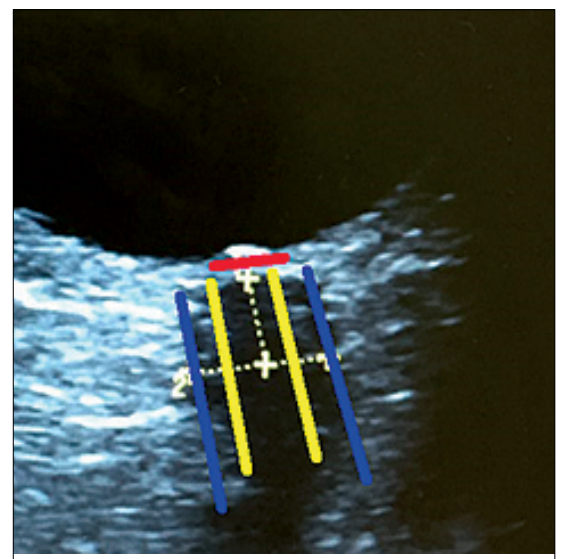

Figura 3. Imagen ultrasonográfica de una medición de la VNO, identificando estructuras (L. roja: Disco óptico; L. amarilla: NO; L. azul : VNO; L. punteada 1: medición de $3 \mathrm{~mm}$ en eje longitudinal NO; L. punteada 2: medición de VNO).

gía ocular traumática, historia de glaucoma u otra patología ocular pueda resultar en un riesgo aumentado de complicaciones locales descritas, así como también secundarios a la mínima presión ejercida por el transductor.

\section{iv) Desarrollo de la técnica}

Dado que no disponemos de alguien entrenado o con experiencia formal en la realización de este examen en nuestra región, nos basamos en la literatura disponible, el apoyo de radiólogos con experiencia en ultrasonido, así como la asesoría electrónica de autores reconocidos en este ámbito.

Según la literatura, Tayal y cols. ${ }^{19}$ estiman que la curva de aprendizaje para la realización del examen, consistiría en 10 casos con 3 resultados anormales, para un ecografista con experiencia; y de al menos 25 casos con 3 anormalidades, para operadores inexpertos $^{7,19}$, convirtiéndola en una técnica 


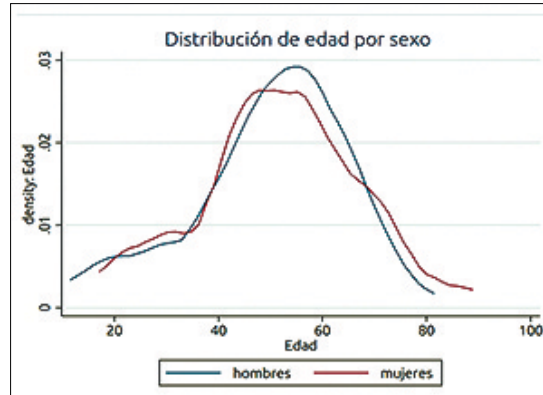

Figura 4. Distribución Edad por sexo.

de fácil entrenamiento.

Por lo que nuestra meta para considerar replicada la técnica, fue mayor a 100 pacientes, siendo las primeras anormalidades corroboradas con el apoyo por correo electrónico, del Dr. Vivek Tayal, reconocido investigador en el tema ${ }^{19}$.

\section{Análisis estadístico}

Para los análisis de resultados se utilizó el software Stata 14,0. A fin de evaluar la calidad de la EVNO en la evaluación de HTEC, construimos una curva ROC usando la medición mediante catéter ventricular externo como referencia, realizando una regresión logística en STATA con los valores de PIC. A partir de esto se calculan los valores de sensibilidad, especificidad y dibuja la curva ROC.

\section{Resultados}

Durante este periodo se instalaron 145 DVE por diversas patologías. Se excluyen del estudio 49 pacientes menores de 18 años. De los restantes 96 casos, 37 fueron excluidos según criterios de exclusión o falta de disponibilidad del operador ecográfico, quedando 59 DVE en un total de 55 pacientes incluidos en el análisis. En total se realizaron 95 mediciones de EVNO. El promedio de edad fue 50 años con una proporción 1,5:1 para hombres y mujeres respectivamente (Figura 4). La principal etiología fue traumática (37\%) seguida de patología tumoral (16\%) e hidrocefalia (15\%), lo que se detalla en la Tabla 1. El promedio global de las mediciones de EVNO y PIC fue de $5,1 \mathrm{~mm}$ y 19 mmhg respectivamente. El promedio de EVNO y PIC para pacientes con HTEC fue $6,1 \mathrm{~mm}$ y $29 \mathrm{mmhg}$, mien-

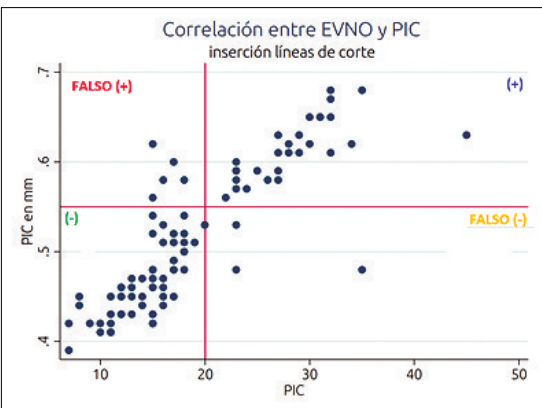

Figura 5. Correlación EVNO y PIC para corte de $5,5 \mathrm{~mm}$. Inserción líneas de corte delimitando verdaderos $y$ falsos positivos y negativos.

tras que para pacientes sin HTEC fue de $4,7 \mathrm{~mm}$ y $14 \mathrm{mmhg}$. En total se identificaron 5 casos falsos (-) y 4 falsos (+). La correlación entre EVNO y PIC está representada en la Figura 5. Los valores obtenidos de sensibilidad y especificidad, para EVNO son de $88 \%$ y $92 \%$ respectivamente, para corte de $5,5 \mathrm{~mm}$ en comparación a una sensibilidad de un $94 \%$ y $81 \%$ para corte de $5,0 \mathrm{~mm}$ (Tabla 2).

Tras el análisis estadístico de las mediciones, se obtuvo una curva de ROC de 0,95 (Figura 6).

Es importante destacar que en dos casos falsos positivos de un mismo paciente, se identificó una compresión extrínseca de ambos nervios ópticos por un quiste aracnoidal complicado, lo que podría explicar la discordancia en relación a la PIC. Es más, tras la resolución quirúrgica de la compresión, se logró una normalización del valor de EVNO, el cual no fue incluido en este análisis por no contar con monitoreo de PIC en el momento de la medición (Figura 7 A-B). Luego de la revisión de imágenes, se identificó otro caso falso positivo, en relación a un artefacto del disco óptico. Se exponen ejemplos de exámenes normal, anormal y artefacto del disco óptico (Figura 8 A-C).

\section{Discusión}

La vaina del nervio óptico es continua con la duramadre encefálica, y de la misma manera el espacio subaracnoideo que contiene el LCR ${ }^{35}$. En 1964, Hayreh y cols., demostraron que cambios en la presión de LCR, podían ser transmitidos a través de los nervios ópticos $^{36}$. Esto ha sido corroborado por estudios clínicos de infusión intratecal,

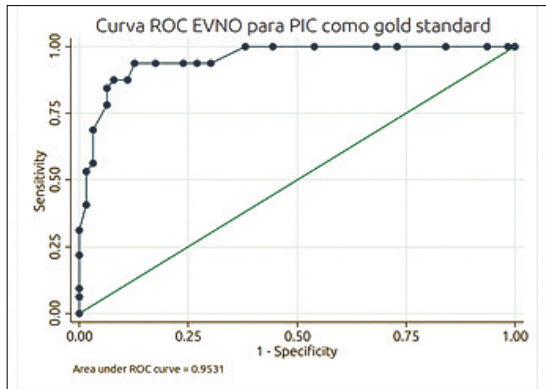

Figura 6. Curva de ROC de EVNO para PIC mediante DVE como referencia.

\section{Tabla 1.}

Distribución según patología

Traumatica $=31 \%(17)$

Tumoral $=16 \%(9)$

Hidrocefalia 15\% (8)

Vascular $=13 \%(7)$

Infecciosa $=11 \%(6)$

Hemorragia primaria $=9 \%(5)$

Isquemica $=5 \%(3)$

Tabla 2.

Valores de sensibilidad, especificidad, valor predictivo positivo y negativo para cortes de $5,5 \mathrm{~mm}$ y $5,0 \mathrm{~mm}$

\section{$5,5 \mathrm{~mm}$}

Sensibilidad $88 \%$

Especificidad 92\%

VPP $85 \%$

VPN $94 \%$

\section{$5,0 \mathrm{~mm}$}

Sensibilidad 94\%

Especificidad 81\%

VPP $71 \%$

VPN $96 \%$

en donde se demuestra que, a diferencia del edema de papila, ocurren variaciones milimétricas en cosa de segundos una vez iniciada la alza de $\mathrm{PIC}^{37,38}$, llegando a una variación máxima en promedio de $1,8 \mathrm{~mm}$ con rango entre 0,7 a 3,1 mm de variación interperso$\mathrm{nal}^{37}$. Lo anterior pudo ser corroborado in situ en nuestra experiencia no repor- 

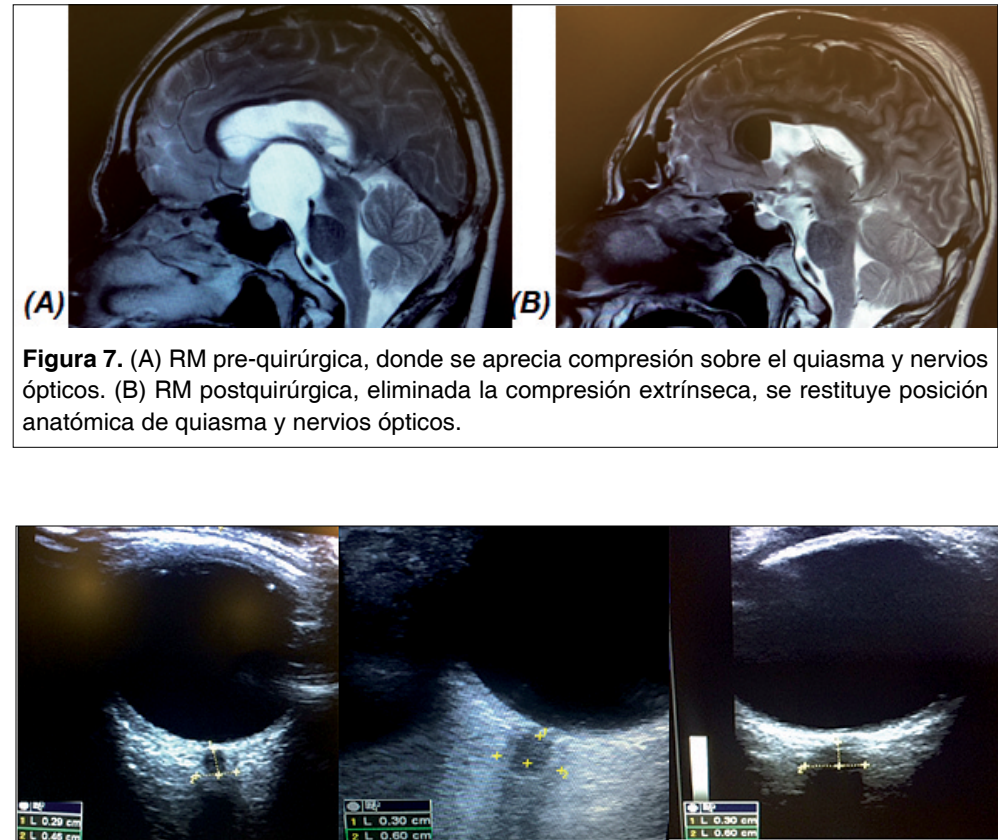

(A)

Figura 8. Imagen de EVNO (A) normal (B) anormal (C) Artefacto del disco óptico.

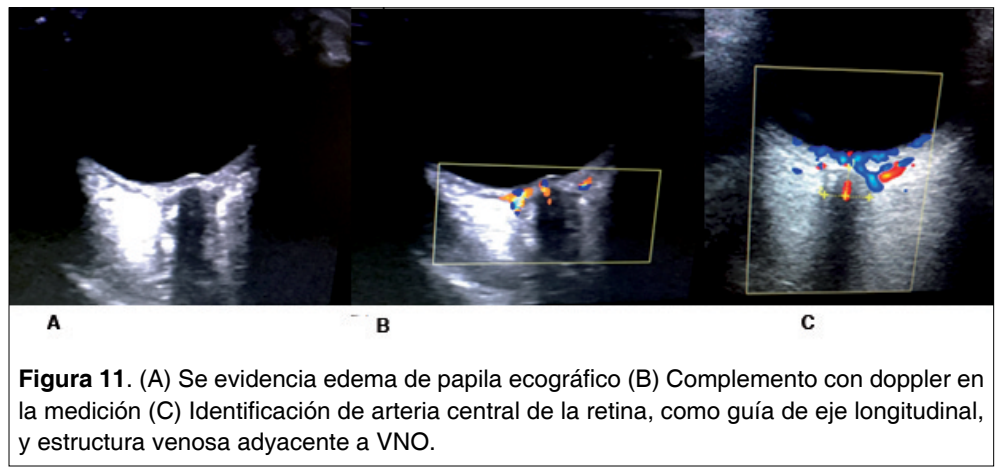

tada, durante la medición de EVNO en pacientes con pseudotumor cerebral, en los cuales se realiza evacuación de LCR, llegando a ser correlativa prácticamente de inmediato. Para determinar el segmento idóneo donde realizar la medición, se han realizado estudios de la microarquitectura de la VNO, destacando la presencia de un trabeculado aracnoideo más laxo y asimétrico presente $3 \mathrm{~mm}$ posteriores al disco óptico, siendo este el punto de mayor distensibilidad y, por lo tanto, el más sensible a cambios de $\mathrm{PIC}^{35,39,40}$. Esto queda representado en la Figura 9.

Como ya se mencionó, la utilidad clínica ya ha sido demostrada en múltiples publicaciones, mostrado una buena correlación con la PIC medida de forma invasiva ${ }^{7,9,10-15,19,25,37-46}$, con resultados publicados en meta-análisis de un $87 \%$ - $90 \%$ de sensibilidad, un $80 \%-85 \%$ de especificidad ${ }^{7,9}$, así como una variabilidad intraobservador e interobservador de $0,2 \mathrm{~mm}$ y 0,2 a $0,3 \mathrm{~mm}$ respectivamente ${ }^{16,17,18}$

El valor de corte es tema de discusión variando entre 4,8 a $6,3 \mathrm{~mm}^{44,45}$, siendo los valores de anormalidad más utilizados entre 5,0 y $5,7 \mathrm{~mm}$ dependiendo del escenario clínico. Esto es sumamente relevante a la hora de interpretar los resultados obtenidos. Como menciona Dubourg en su meta-análisis, el no diagnóstico de un síndrome de HTEC puede llevar a consecuencias catastróficas, por lo cual los clínicos deben abogar por una mayor sensibi-

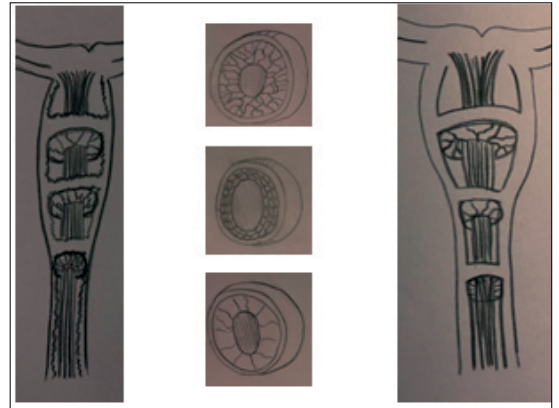

Figura 9. Diagrama explicativo de dilatación de la VNO. (Adaptado de Killer HE et al).

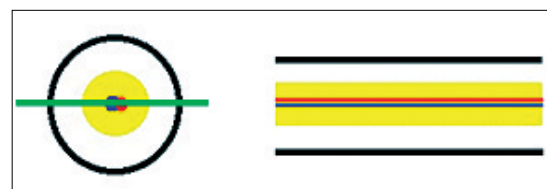

Figura 10. Esquema de corte ultrasonográfico con apoyo doppler.

lidad y valor predictivo negativo, como herramienta para descartar el diagnóstico, en escenarios donde la pesquisa es lo primordial ${ }^{7}$, como por ejemplo los servicios de urgencias. Por otra parte, en escenarios controlados de HTEC conocida o altamente probable, cobra mayor relevancia la especificidad, herramienta para confirmar el diagnóstico, a fin de poder controlar o guiar un tratamiento en un contexto clínico conocido, como por ejemplo un pseudotumor cerebral. Por nuestra parte optamos por utilizar $5,5 \mathrm{~mm}$ debido su alta especificidad en un escenario neuroquirúrgico, con resultados concordantes a los trabajos de Bäuerle, Soldatos y Amini ${ }^{15,25,46}$. Es importante destacar que existe variabilidad de valores reportados en individuos sanos, dependiendo del país de origen, siendo 5,1 $\mathrm{mm}$ para China ${ }^{46}, 3,7 \mathrm{~mm}$ para Canadá $47,3,6 \mathrm{~mm}$ para Griegos ${ }^{15}$ y 4,4 mm en Bangladesh ${ }^{50}$, no existiendo datos al respecto en la población chilena, por lo que no contamos con información respecto a la media en nuestra población. Dentro del análisis de los falsos positivos identificados, se debe considerar importancia de descartar compresión extrínseca de las VNO, a la hora de considerar el examen como compatible con HTEC. También mencionar la relevancia reconocer la presencia de un artefacto ecogénico, como lo es la sombra acústica ocasionada por el disco óptico, ya publicada en la literatura ${ }^{49}$. 
Con el desarrollo de la técnica, confirmamos la gran utilidad del complemento de la EVNO con técnica doppler, lo cual permite identificar la dirección y eje central de la VNO (Figura 10), así como esclarecer los márgenes laterales, logrando diferenciar la superposición de estructuras venosas frecuentemente aledañas (Figura $11 \mathrm{~A}-\mathrm{C}$ ).

Para finalizar, respecto al fondo de ojo bajo dilatación pupilar, la sensibilidad y especificad descrita para papiledema en pacientes con HTEC, se encuentra entre $83 \%$ a $100 \%$ y 88 a $96 \%$ respectivamente ${ }^{30}$, si bien varios trabajos demuestran que estos valores son variables dependiendo del grado de especialización médica, los valores más bajos de sensibilidad y especificidad son $84,5 \%$ y $59 \%$ respectivamente, correspondientes a los médicos de familia ${ }^{31}$. A esto se deben sumar las limitaciones propias de la técnica que, además de contar con el oftalmoscopio, se debe realizar bajo dilatación pupilar, colaboración del paciente y escasa aplicabilidad en casos agudos, considerando la temporalidad en la génesis del edema papilar.

Este reporte constituye el primero publicado en nuestro país, para la validación de esta técnica no invasiva en nuestra realidad.

\section{Conclusión}

La EVNO es una técnica no invasiva, reproducible, sensible y específica para la detección de HTEC, concordante con lo publicado en la literatura, siendo este el primer reporte para su validación y difusión en Chile.

En opinión del autor, esta técnica no debe reemplazar el monitoreo de PIC invasivo cuando este sea indicado, sino más bien destacar el gran potencial de esta técnica más allá del neurointensivo, la rápida curva de aprendizaje, la amplia disponibilidad, la rapidez de su realización, su bajo costo, sus características no invasivas y la factibilidad de realizarla sin movilizar al paciente, la convierten en una herramienta útil a la hora de evaluar pacientes en unidades de urgencia y en la práctica clínica co- tidiana, tanto para especialistas como no especialistas. También destacar su potencial utilidad como método de seguimiento en patologías que cursen riesgo de HTEC y pacientes post operados neuroquirúrgicos.

Trabajo de Ingreso LX Congreso Chileno de Neurocirugía, Pucón 2017.

Agradecimientos: A mi querida esposa e hijo, Francisca Guerrero y Matías García, por su apoyo incondicional. A mis colegas por su constante apoyo, enseñanzas y ayuda en la realización de este trabajo: Dr. Leonidas Quintana, Dr. Camilo Arriagada, Dr. Filadelfo Norambuena, Dr. Benjamín Abarca, Dr. Cristian Salazar, Dr. Felipe Sfeir, Dr. Pablo Carmona, Dr. José Luis Cuevas, Dr. Jorge Cerda, Dra. Lorena Toro, Dr. Cristian Toloza, Dr. Yuri Carvajal, Dr. Vivek Tayal.

Recibido: 9 de septiembre de 2018 Aceptado: 5 de mayo de 2019

\section{Referencias}

1. Adams RA, Ropper AH. Principles of neurology, 6th, McGraw Hill, New York 1997.

2. Smith E, Amin-Hanjani S. Evaluation and management of elevated intracranial pressure in adults. UpTodate jul 10, 2013.

3. Brain Trauma Fundation Guidelines 2010. Inhospital Sever TBI Guidelines. VII Intracranial Pressure Monitoring Technology.

4. Raboel PH, Bartek J Jr, Andresen M, Bellander BM, Romner B. Intracranial Pressure Monitoring: Invasive versus Non-Invasive Methods-A Review. Crit Care Res Pract. 2012;2012:950393. doi: 10.1155/2012/950393. Epub 2012 Jun 8.

5. DD Binz, LG. Toussaint III, and JA. Friedman. Hemorrhagic complications of ventriculostomy placement: a meta-analysis. Neurocrit Care 2009 , vol. 10 , no. 2 , pp. 253-256.

6. Lozier AP, Sciacca RR, Romagnoli MF, Connolly ES Jr. Ventriculostomy-related infections: a critical review of the literature. Neurosurgery. 2002 Jul;51(1):170-81; discussion 181-2.

7. Dubourg J, Javouhey E, Geeraerts T, Messerer M, Kassai B. Ultrasonography of optic nerve sheath diameter for detection of raised intracranial pressure: a systematic review and meta-analysis. Intensive Care Med. 2011 Jul;37(7): 1059-68.

8. Rosenberg JB, Shiloh AL, Savel RH, Eisen LA. Non-invasive methods of estimating intracranial pressure. Neurocrit Care. 2011: Dec;15(3): 599-608.

9. Moretti R, Pizzi B. Ultrasonography of the optic nerve in neurocritically ill patients. Acta Anaesthesiol Scand. 2011 Jul;55(6):644-52. doi: 10.1111/j.1399-6576.2011.02432.x. Epub 2011 Apr 4.

10. Geeraerts T, Launey Y, Martin L,Pottecher J, Vigue B, Duranteau J,Benhamou D (2007) Ultrasonographyof the optic nerve sheath may be useful for detecting raised intracranial pressure after severe brain injury. Intensive Care Med 33: 1704-1711.

11. Geeraerts T, Merceron S, Benhamou D, Vigue B, Duranteau J (2008) Noninvasive assessment of intracranial pressure using ocular sonography in neurocritical care patients. Intensive Care Med 34: 2062-2067.

12. Kimberly HH, Shah S, Marill K, NobleV (2008). Correlation of optic nerve sheath diameter with direct measurement of intracranial pressure

13. Moretti R, Pizzi B (2009) Optic nerve ultrasound for detection of intracranial hypertension in intracranial hemorrhage patients: confirmation of previous findings in a different patient population. J Neurosurg Anesthesiol 21: 16-20.

14. Moretti R, Pizzi B, Cassini F, Vivaldi N (2009). Reliability of optic nerve ultrasound for the evaluation of patients with spontaneous intracranial hemorrhage. Neurocrit Care 11: 406-410.

15. Soldatos T, Karakitsos D, Chatzimichail K, Papathanasiou M, Gouliamos A, Karabinis A (2008) Optic nerve sonography in the diagnostic evaluation of adult brain injury. Crit Care 12:R67.

16. Bäuerle J, Lochner P, Kaps M, Nedelmann M. Intra and interobsever reliability of sonographic assessment of the optic nerve sheath diameter in healthy adults. J Neuroimaging. 2012 Jan;22(1):42-5. doi: 10.1111/j.1552-6569.2010.00546.x.

17. Bäuerle J, Schuchardt F, Schroeder L, Egger K, Weigel M, Harloff A. Reproducibility and accuracy of optic nerve sheath diameter assessment using ultrasound compared to magnetic resonance imaging. BMC Neurol. 2013: Dec 1;13(1): 187.

18. Ballantyne SA, O'Neill G, Hamilton R, Hollman AS (2002) Observer variation in the sonographic measurement of optic nerve sheath dia- 
meter in normal adults. European journal of ultrasound: official journal of the European Federation of Societies for Ultrasound in Medicine and Biology 15: 145-149.

19. Tayal VS, Neulander M, Norton HJ, Foster T, Saunders T, Blaivas M. mergency department sonographic measurement of optic nerve sheath diameter to detect findings of increased intracranial pressure in adult head injury patients. Ann Emerg Med. $2007 ; 49: 508-514$.

20. Patel HC, Bouamra O, Woodford M, King AT, Yates DW, Lecky FE. Trends in head injury outcome from 1989 to 2003 and the effect of neurosurgical care: an observational study. Lancet 2005; 366: 1538-44.

21. Loy CT, Irwig L. Accuracy of diagnostic tests read with and without clinical information: a systematic Review.

22. Gerlach H, Toussaint S. Sensitive, specific, predictive statistical basics: how to use biomarkers. Crit Care Clin. 2011 Apr

23. Akobeng AK. Understanding diagnostic tests 2: likelihood ratios, pre- and post-test probabilities and their use in clinical practice. Acta Paediatr. 2007 Apr. 1; 96(4): 48

24. Shapiro DE. The interpretation of diagnostic tests. Stat Methods Med Res. 1999: Jun;8(2): 113-134.

25. Bäuerle J, Nedelman N. B-mode sonography of the optic nerve in neurological disorders with altered intracranial pressure. Perspectives in Medicine (2012) 1, 404-407.

26. U.S. Food and Drug Administration. Guidance for Industry and FDA Staff Information for Manufacturers Seeking Marketing Clearance of Diagnostic Ultrasound Systems and Transducers. Silver Spring, MD: U.S. Food and Drug Administration, 2008.

27. Barnett SB. World Federation for Ultrasound in Medicine and Biology (WFUMB) Symposium on safety of ultrasound in medicine: conclusions and recommendations on thermal and non-thermal mechanisms for biological effects of ultrasound. Ultrasound Med Biol 1998; 24: $1-55$.

28. Bergès $\mathrm{O}$, Koskas $\mathrm{P}$, Lafitte F, Piekarski JD. Sonography of the eye and orbit with a multipurpose ultrasound unit. J Radiol. 2006 Apr;87(4 Pt 1): 345-53.

29. Maude RR, Amir Hossain M, Hassan MU, Osbourne S, Sayeed KL, Karim MR, Samad R, Borooah S, Dhillon B, Day NP, Dondorp AM, Maude RJ. Transorbital sonographic evaluation of normal optic nerve sheath diameter in healthy volunteers in bangladesh. PLoS One 2013 Dec 2;8(12):e81013. doi: 10.1371/journal.pone.0081013.

30. Frisen L. Swelling of the optic nerve head: a staging scheme.J Neurol Neurosurg Psychiatry. 1982; 45:13-8.

31. Johnson LN, Hepler RS, Bartholomew MJ. Accuracy of papilledemaand pseudopapilledema detection: a multispecialty study. J Fam Pract. 1991; 33: 381-6.

32. Journal of neurotrauma volume 24, supplement 1, 2007.

33. Marshall LF, Marshall SB, Klauber MR, Clark MvB, Eisenberg HM, Jane JA, Luerssen TG, Marmarou A, Foulkes MA: A new classification of head injury based on computerized tomography. J Neurosurg 75: S14-S20, 1991.

34. Maas Al, Hukkelhoven CW, Marshall LF, Steyerberg EWPrediction of outcome in traumatic brain injury with computed tomographic characteristics: a comparison between the computed tomographic classification and combinations of computed tomographic predictors. 2005 Dec;57(6):1173-82; discussion 1173-82.

35. Killer HE, Laeng HR, Flammer J, Groscurth P. Architecture of arachnoid trabeculae, pillars, and septa in the subarachnoid space of the human optic nerve: Anatomy and clinical considerations. Br J Ophthalmol. 2003; 87: 777-81.

36. Hayreh SS. Pathogenesis of oedema of the optic disc (pappiloedema).A preliminary report. Br J Ophthalmol. 1964 ;48: $522-43$.

37. Hansen $\mathrm{HC}$, Helmke K. Validation of the optic nerve sheath response to changing cerebrospinal fluid pressure: ultrasound findings during intrathecal infusion tests. J Neurosurg. $1997 \mathrm{Jul} ; 87(1)$ : 34-40.

38. Geeraerts T, Duranteau J, Benhamou D. Ocular sonography in patients with raised intracranial pressure: The papilloedema revisited. Crit Care. 2008; 12: 150

39. Helmke K, Hansen HC (1996) Fundamentals of transorbital sonographic evaluation of optic nerve sheath expansion under intracranial hypertension ii. Patient study. Pediatr Radiol 26: 706-710.

40. Hansen H-C, Lagrèze W, Krueger O, Helmke K. Dependence of the optic nerve sheath diameter on acutely applied subarachnoidal pressure - an experimental ultrasound study. Acta Ophthalmologica. 2011; 89(6).

41. Robba C, Cardim D, Tajsic T, et al. Ultrasound non-invasive measurement of intracranial pressure in neurointensive care: A prospective observational study. PLOS Medicine. 2017;14(7). doi:10.1371/journal.pmed.1002356.

42. Frumin E, Schlang J, Wiechmann W, et al. Prospective Analysis of Single Operator Sonographic Optic Nerve Sheath Diameter Measurement for Diagnosis of Elevated Intracranial Pressure. Western Journal of Emergency Medicine. 2014; 15(2): $217-220$.

43. Raffiz M, Abdullah JM. Optic nerve sheath diameter measurement: a means of detecting raised ICP in adult traumatic and non-traumatic neurosurgical patients. The American Journal of Emergency Medicine. 2017;35(1):150-153. doi:10.1016/j.ajem.2016.09.044.

44. Rajajee V, Vanaman M, Fletcher JJ, Jacobs TL. Optic Nerve Ultrasound for the Detection of Raised Intracranial Pressure. Neurocritical Care. 2011; 15(3): 506-515

45. Sekhon MS, Mcbeth P, Zou J, et al. Association Between Optic Nerve Sheath Diameter and Mortality in Patients with Severe Traumatic Brain Injury. Neurocritical Care. 2014; 21(2): 245-252.

46. Amini $\mathrm{A}$, Kariman $\mathrm{H}$, Dolatabadi $\mathrm{AA}$, et al. Use of the sonographic diameter of optic nerve sheath to estimate intracranial pressure. The American Journal of Emergency Medicine. 2013;31(1):236-239. doi:10.1016/j.ajem.2012.06.025

47. Chen H, Ding GS. \& Zhao YC, et al. Ultrasound measurement of optic nerve diameter and optic nerve sheath diameter in healthy Chinese adults. BMC Neurol. 7(15), 106 (2015).

48. Goeres P, Zeiler FA, Unger B, Karakitsos D. \& Gillman LM. Ultrasound assessment of optic nerve sheath diameter in healthy volunteers. J Crit Care. 31(1), 168-171 (2016).

49. Copetti, R, \& Cattarossi L.(2009). Optic nerve ultrasound: artifacts and eal images. Intensive Care Medicina, 35(8); 1488-1489.

50. Maude RR, Hossain MA, Hassan MU, Osbourne S, Sayeed KL, Karim MR, Maude RJ. (2013). Transorbital Sonographic Evaluation of Normal Optic Nerve Sheath Diameter in Healthy Volunteers in Bangladesh. PLoS ONE,8(12).

\section{Correspondencia a:}

Dr. Julio García Molina

Neurocirujano, Hospital Puerto Montt, Chile.

drjuliogarcianeuro@ hotmail.com 